\title{
Brain injury, endothelial injury and inflammatory markers are elevated and express sex-specific alterations after COVID-19
}

Jude Savarraj ${ }^{1 *}$ (D) Eun S. Park' ${ }^{1}$ Gabriela D. Colpo ${ }^{4}$, Sarah N. Hinds ${ }^{1}$, Diego Morales ${ }^{4}$, Hilda Ahnstedt ${ }^{4}$, Atzhiry S. Paz ${ }^{1}$, Andres Assing ${ }^{1}$, Fudong Liu ${ }^{4}$, Shivanki Juneja ${ }^{4}$, Eunhee Kim', Sung-min Cho ${ }^{3}$, Aaron M. Gusdon ${ }^{1}$, Pramod Dash², Louise D. McCullough ${ }^{4}$ and H. Alex Choi ${ }^{*}$

\begin{abstract}
Objective: Although COVID-19 is a respiratory disease, all organs can be affected including the brain. To date, specific investigations of brain injury markers (BIM) and endothelial injury markers (EIM) have been limited. Additionally, a male bias in disease severity and mortality after COVID-19 is evident globally. Sex differences in the immune response to COVID-19 may mediate this disparity. We investigated BIM, EIM and inflammatory cytokine/chemokine (CC) levels after COVID-19 and in across sexes.

Methods: Plasma samples from 57 subjects at $<48$ h of COVID-19 hospitalization, and 20 matched controls were interrogated for the levels of six BIMs-including GFAP, S100B, Syndecan-1, UCHLI, MAP2 and NSE, two EIMs -including sICAM1 and sVCAM1. Additionally, several cytokines/chemokines were analyzed by multiplex. Statistical and bioinformatics methods were used to measure differences in the marker profiles across (a) COVID-19 vs. controls and (b) men vs. women.

Results: Three BIMs: MAP2, NSE and S100B, two EIMs: sICAM1 and SVCAM1 and seven CCs: GRO IL10, sCD40L, IP10, IL1Ra, MCP1 and TNFa were significantly $(p<0.05)$ elevated in the COVID-19 cohort compared to controls. Bioinformatics analysis reveal a stronger positive association between BIM/CC/EIMs in the COVID-19 cohort. Analysis across sex revealed that several BIMs and CCs including NSE, IL10, IL15 and IL8 were significantly $(p<0.05)$ higher in men compared to women. Men also expressed a more robust BIM/ EIM/CC association profile compared to women.
\end{abstract}

Conclusion: The acute elevation of BIMs, CCs, and EIMs and the robust associations among them at COVID-19 hospitalization are suggestive of brain and endothelial injury. Higher BIM and inflammatory markers in men additionally suggest that men are more susceptible to the risk compared to women.

Keywords: Brain injury, COVID-19, SARS-CoV-2, Inflammation, Endothelial injury, Sex differences

*Correspondence: Jude.p.savarraj@uth.tmc.edu; Huimahn.A.Choi@uth.tmc. edu

${ }^{1}$ Departent of Neurosurgery, McGovern Medical School, The University of Texas Health Science Center at Houston, 6431 Fannin St, Houston, TX 77030, USA

Full list of author information is available at the end of the article

\section{Introduction}

Neurologic complications after COVID-19 have been reported in both the acute and chronic period [1,2]. Neurologic manifestations including ischemic and hemorrhagic stroke have been reported after COVID-19. But neurologic manifestations without clear etiology original author(s) and the source, provide a link to the Creative Commons licence, and indicate if changes were made. The images or other third party material in this article are included in the article's Creative Commons licence, unless indicated otherwise in a credit line to the material. If material is not included in the article's Creative Commons licence and your intended use is not permitted by statutory regulation or exceeds the permitted use, you will need to obtain permission directly from the copyright holder. To view a copy of this licence, visit http://creativecommons.org/licenses/by/4.0/. The Creative Commons Public Domain Dedication waiver (http://creativeco mmons.org/publicdomain/zero/1.0/) applies to the data made available in this article, unless otherwise stated in a credit line to the data. 
including encephalopathy, pain, chronic fatigue and cognitive dysfunction have also been reported demonstrating the wide impact of COVID-19 on the nervous system [3-8]. Human coronaviruses $(\mathrm{HCoV})$ are neuroinvasive and neurotropic by nature and may contribute to both short- and long-term neurological disorders [9-11]. SARS-CoV (of the SARS epidemic in early 2000s) has been shown to affect the CNS [12] and viral strains have been identified in post-mortem human brain tissue $[12,13]$. Our understanding of the effect of COVID19 on brain injury is only beginning. COVID-19 triggers multiple pathological mechanisms-including an acute inflammatory response and endotheliopathy-and the relationship of these mechanisms with brain injury is unclear.

Sex differences in COVID-19 are prominent, with men having more severe disease, with higher hospitalization and mortality rates, despite similar rates of infection-an effect seen globally $[14,15]$. Sex differences in immune response may underlie the increase in mortality seen in men during the acute disease course $[16,17]$. It is uncertain whether the increase in peripheral inflammation seen in accompanied by an increase in brain injury markers as well.

We investigated brain injury markers (BIMs), endothelial injury markers (EIMs) and inflammatory markers in COVID-19 patients in the acute phase $(<48 \mathrm{~h})$ after hospitalization. Several BIMs have been reported as surrogate markers of neuronal and astrocytic injury in nonneurological diseases like HIV [18-20], cardiac arrest [21-23] and sepsis [24]. Among them, we measured six validated BIMs including glial fibrillary acidic protein (GFAP), neuron-specific enolase (NSE), S100B, ubiquitin carboxyl-terminal hydrolase isozyme L1 (UCHL1), Syndecan-1 and microtubule-associated protein 2 (MAP 2) in plasma drawn from hospitalized COVID-19 subjects and matched controls. In addition to BIMs, we measured two EIMs [including Intercellular Adhesion Molecule 1 (ICAM-1) and Vascular Cell Adhesion Molecule 1 (VCAM-1)] and cytokine/chemokine markers (CCs) of systemic inflammation to investigate if endothelial injury and inflammation are associated with BIMs. The levels of the markers were measured by ELISA and multiplex assays, and the association between COVID-19 and controls were characterized using statistical and bioinformatics approaches. The differences in the levels of markers between men and women were investigated.

\section{Methods}

\section{Study population and patient inclusion and exclusion criteria}

This is a prospective study of COVID-19 patients admitted and hospitalized at the Memorial Herman Hospital
System in Houston, Texas, USA. Inclusion criteria were laboratory-confirmed SARS-CoV-2 infection by real-time polymerase chain reaction, written informed consent from the patient or surrogate, and age $\geq 18$ years. Exclusion criteria were inability to complete long-term followup, severe functional disabilities before hospital admission for COVID-19 [defined by pre-admission modified Rankin Score (mRS) [25] $>1]$, history of pulmonary complications (including resection and transplant), pre-existing systemic diseases which would impact long-term outcomes (including stroke, myocardial infarction, pulmonary disease requiring home oxygen, chronic renal failure necessitating hemodialysis and malignancy), documented neurologic and psychiatric disorders, prisoners and pregnant women. Patients were categorized as mild (nasal cannula with $<5 \mathrm{~L}$ of $\mathrm{O}_{2}$ and $<5$ days of hospitalization), moderate (nasal cannula with $>5 \mathrm{~L}$ of $\mathrm{O}_{2}$ or heat high-flow cannula and $>5$ days of hospitalization) and severe (on ventilator or expired). Plasma samples from 24 non-neurological subjects who were enrolled at the UT Physician Cardiology clinic were used as controls. The controls were matched to the COVID-19 cohort for age, sex and co-morbidities (including diabetes and hypertension). This study was approved by the 'Institutional Review Board' (IRB No: HSC MH-17-0452) at The University of Texas Health Science Center at Houston, Houston, Texas.

\section{Samples}

Blood was drawn by venipuncture, collected into sterile vacutainers, and immediately placed on ice. For processing of plasma, the tubes were centrifuged at $1200 \times g$ for $10 \mathrm{~min}$ at $4{ }^{\circ} \mathrm{C}$ followed by a second centrifugation at $10,000 \times g$ for $10 \mathrm{~min}$ at $4{ }^{\circ} \mathrm{C}$ to generate plateletpoor plasma. Plasma were then aliquoted and stored at $-80^{\circ} \mathrm{C}$ until the samples were analyzed.

\section{ELISA and multiplex analysis}

BIMs were measured using the following ELISA kits: GFAP (Cat No: NS830, Sigma-Aldrich), NSE (Cat No: 420-85, IBL America), S100B (Cat No: EZHS100B-33K, MilliporeSigma), UCH-L1 (EH475RB, ThermoFisher), syndecan-1(RAB0736-1KT, MilliporeSigma) and MAP2 (EKU05950, BIOMATIK). The EIMs (including ICAM-1 and VCAM-1) were measured using a premixed multiplex assay (Cat. \#HCVD2MAG-67K, MilliporeSigma). Systemic levels of CCs were measured using a 38-plex premixed immunological multiplex assay (HCYTMAG60K-PX38, MilliporeSigma, Billerica, MA). CCs included CD40L, EGF, Eotaxin/CCL11, FGF-2, Flt-3 ligand, Fractalkine, G-CSF, GM-CSF, GRO, IFN- $\alpha 2$, IFN- $\gamma$, IL- $1 \alpha$, IL-1 $\beta$, IL-1ra, IL-2, IL-3, IL-4, IL-5, IL-6, IL-7, IL-8, IL-9, IL-10, IL-12 (p40), IL-12 (p70), IL-13, IL-15, IL-17A, IP-10, MCP-1, MCP-3, MDC (CCL22), MIP-1 $\alpha$, MIP-1 $\beta$, 
TGF- $\alpha$, TNF- $\alpha$, TNF- $\beta$ and VEGF. All experiments were performed according to manufacturer's protocol. The levels of all markers are reported as $\mathrm{pg} / \mathrm{ml}$.

\section{Statistical analysis}

Descriptive statistics were calculated for demographic variables and protein levels in control and COVID-19 subjects. To describe differences in demographics, $X^{2}$ test, Fisher's exact test, Student's $t$-test, and the MannWhitney $U$ test were used where appropriate. The Mann-Whitney $\mathrm{U}$ test was used to test for differences in protein levels across different groups A $p$-value of $\leq 0.05$ was considered statistically significant (two-tailed). The levels of the markers are reported as mean \pm standard error (SE). All statistical analyses were performed using open-source software packages in R (v3.1.3).

\section{Bioinformatics and chord diagrams}

To elucidate the relationship between the BIMs, EIMs and CCs, we employed the chord diagram technique that is useful in visualizing relationships in multi-dimensional data. A chord diagram is a graphical method that is typically used to display inter-relationships between large numbers of variables. In this study, the variables are the markers that were investigated (the CCs, BIMs and the EIMs) and the relationship between them is the Pearson's correlation coefficient (PCC). Each marker distribution was first normalized via Box-Cox transformations [26] and the PCCs were calculated ( $\mathrm{R}$ v3.1.3) for each pair of the markers. This resulted in a large correlation matrix with each entry in the matrix representing a PCC between corresponding markers. We retained only the significant $(p<0.05)$ PCCs and constructed the chord diagrams. In the chord diagram, we arranged each marker sub-type (i.e., CCs, BIMs and EIMs) as an outer sector that were color-coded (green for CCs, red for BIMs and dark blue for EIMs) to maintain visualization consistent with other figures presented. The variables (the CCs, BIMs and the EIMs) were represented by the inner-sectors and the length of the inner-sectors was mapped to the number of significant PCCs that the corresponding variable had with other variables in the dataset. A longer inner-sector denotes that the corresponding variable had a high number of PCCS with other markers. The random-skewers method [27] was used to test for statistical differences across the chord diagrams. The $\mathrm{R}$ package circlize [28] was used to construct the chord diagrams.

\section{Modified Rankin assessment}

The functional status of the patient was quantified using the modified Rankin's scale (mRS) which is a $0-6$ point grading scale of the patient's functional status [29]. '0': no deficits, ' 1 ': presence of symptoms but no significant disability, '2': a slight disability but independent, ' 3 ': moderate disability requiring some help but able to walk without assistance, '4': a moderately severe disability and unable to walk and attend to bodily needs without assistance, ' 5 ': a severe disability where the patient requires constant nursing care and attention and, ' 6 ': being dead. For the purposes of this study, we dichotomized patients into two groups; good $(\mathrm{mRS} \leq 3)$ and poor $(\mathrm{mRS} \geq 4)$ outcomes. The mRS at discharge was assessed.

\section{Results}

\section{Patient characteristics}

Samples from 57 subjects and 20 control subjects were included in the study. Demographics and clinical outcomes of the COVID-19 subjects and controls are presented (Table 1). There were no significant differences in age and sex distribution between the two groups. The COVID-19 cohort had a significantly more Hispanic subjects compared to the control ( $56 \%$ vs. $10 \%, p<0.01$ ). There were no differences in the history of hypertension, diabetes and coronary heart disease between the two groups. However, the COVID-19 group had more obese subjects compared to controls ( $49 \%$ vs. $20 \%, p=0.03$ ).

\section{BIMs after COVID-19 hospitalization}

At $<48 \mathrm{~h}$ hospitalization for of COVID-19, the mean levels of MAP2 ( $68 \pm 7.5$ vs. $26 \pm 3.9, \mathrm{pg} / \mathrm{ml}, p<0.01)$, NSE $(16.9 \pm 3.2$ vs. $4.9 \pm 0.59, \mathrm{pg} / \mathrm{ml}, p<0.01)$ and $\mathrm{S} 100 \mathrm{~B}$ $(113 \pm 16$ vs. $43 \pm 7, \mathrm{pg} / \mathrm{ml}, p<0.01)$ were significantly higher compared to controls (Fig. 1A). In the COVID-19 cohort, the average levels of MAP2, NSE and S100B were $160 \%, 245 \%$ and $162 \%$ higher than controls.

Table 1 Demographics and past medical history

\begin{tabular}{lccc}
\hline Variables & $\begin{array}{c}\text { Control } \\
\mathbf{N = 2 0}\end{array}$ & $\begin{array}{c}\text { COVID-19 } \\
\mathbf{N = 5 7}\end{array}$ & $\boldsymbol{p}$ \\
\hline $\begin{array}{l}\text { Demographics } \\
\text { Age (mean, sd) }\end{array}$ & $61(15)$ & $53(18)$ & 0.07 \\
Sex, female $(n, \%)$ & $11(55)$ & $24(42)$ & 0.4 \\
Race $(n, \%)$ & & & \\
$\quad$ White & $16(80)$ & $35(61)$ & 0.17 \\
$\quad$ African-American & $3(15)$ & $15(26)$ & 0.37 \\
$\quad$ Others & $1(5)$ & $7(12)$ & 0.67 \\
Ethnicity, Hispanic $(n, \%)$ & $2(10)$ & $32(56)$ & $<\mathbf{0 . 0 1}$ \\
Past medical history & & & \\
Hypertension $(n, \%)$ & $5(25)$ & $24(42)$ & 0.28 \\
Diabetes $(n, \%)$ & $4(20)$ & $24(42)$ & 0.16 \\
CAD $(n, \%)$ & $5(25)$ & $7(12)$ & 0.16 \\
Obesity $(n, \%)$ & $4(20)$ & $28(49)$ & $\mathbf{0 . 0 3}$ \\
\hline
\end{tabular}

$p$-values $<0.05$ are shown in bold

$S D$ standard deviation, $C A D$ coronary artery disease 


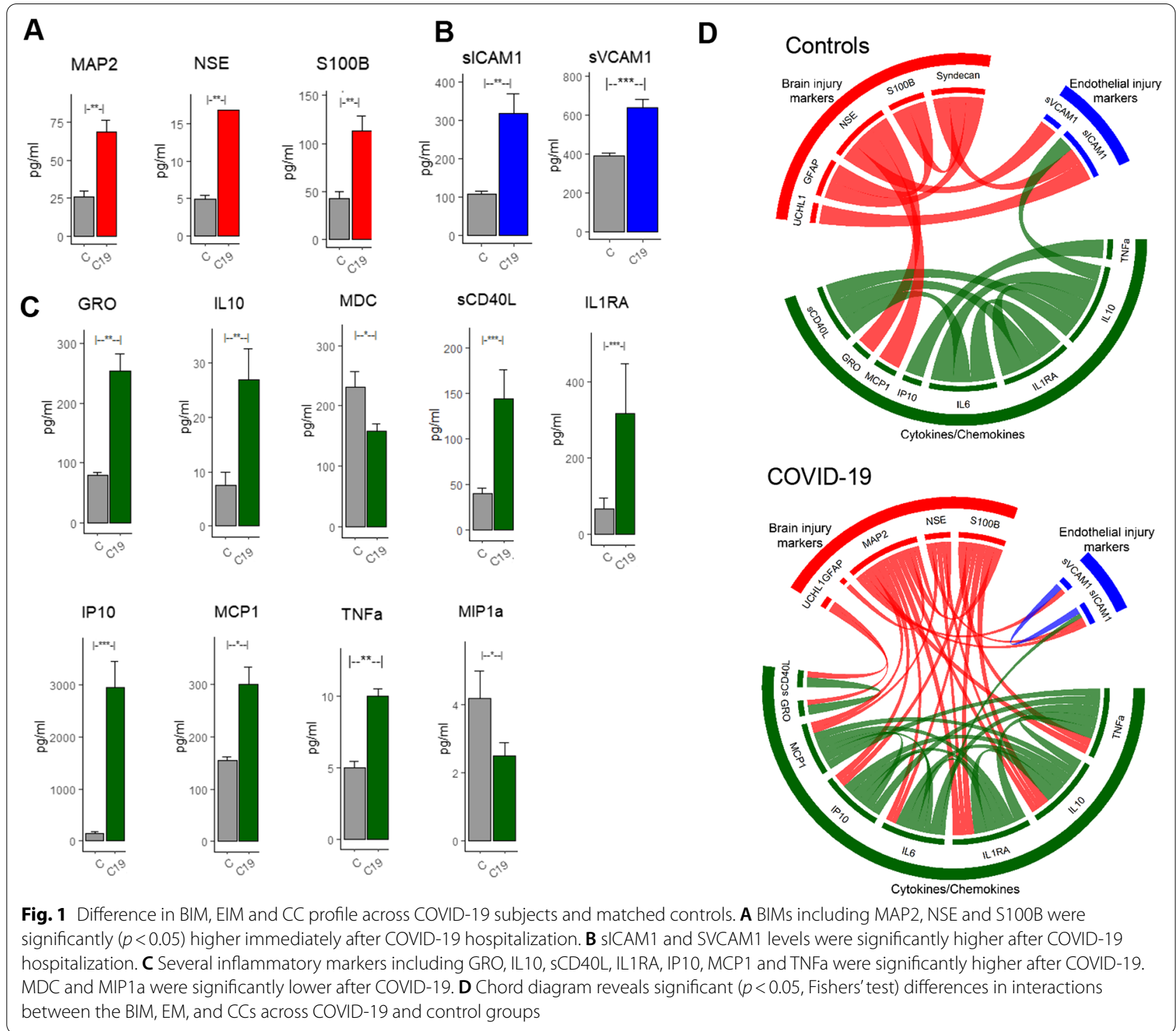

EIMs and inflammatory CCs after COVID-19 hospitalization At the acute phase $(<48 \mathrm{~h})$, the levels of the EIMs and most CCs were significantly higher in the COVID-19 cohort compared to controls. EIMs including SICAM1 $(317 \pm 51$ vs. $108 \pm 7.5, \mathrm{pg} / \mathrm{ml}, p<0.001)$ and sVCAM1 $(637 \pm 45$ vs. $390 \pm 16, \mathrm{pg} / \mathrm{ml}, p<0.01)$ were significantly higher in COVID-19 compared to controls (Fig. 1B). Of the $38 \mathrm{CCs}$ investigated, 7 CCs were significantly higher and $2 \mathrm{CCs}$ were significantly lower in COVID-19 (Fig. 1C). The 7 CCs that were significantly higher were GRO $(254 \pm 29$ vs. $79 \pm 5.19, \mathrm{pg} / \mathrm{ml}$, $p<0.01)$, IL10 (27 \pm 5.7 vs. $7.4 \pm 2.5, \mathrm{pg} / \mathrm{ml}, p<0.01)$, $\mathrm{sCD} 40 \mathrm{~L} \quad(144 \pm 32$ vs. $40 \pm 6, \mathrm{pg} / \mathrm{ml}, \quad p<0.0001)$, $\operatorname{IL} 1 \operatorname{Ra}(318 \pm 131$ vs. $68 \pm 28, \mathrm{pg} / \mathrm{ml}, \quad p<0.01), \quad$ IP10 $(2952 \pm 512$ vs. $148 \pm 20, \mathrm{pg} / \mathrm{ml}, \quad p<0.001), \quad \mathrm{MCP} 1$
$(300 \pm 34$ vs. $154 \pm 8, \mathrm{pg} / \mathrm{ml}, \quad p<0.05)$ and TNFa $(10 \pm 0.5$ vs. $5 \pm 0.4, \mathrm{pg} / \mathrm{ml}, p<0.01)$. The two CCs that were significantly lower in the COVID-19 cohort were $\mathrm{MDC}(158 \pm 12$ vs. $231 \pm 26, \mathrm{pg} / \mathrm{ml}, p<0.05)$ and MIP1a $(2.5 \pm 0.4$ vs. $4.2 \pm 0.8, \mathrm{pg} / \mathrm{ml}, p<0.05)$. The levels of all non-significant BIMs, CCs and EIMs in Control vs. COVID-19, male vs. female COVID-19 subjects and across severity are tabulated in Tables S1, S2 and S3 in the Additional file 1.

\section{The association with inflammatory cytokines and COVID-19}

Bioinformatics (chord diagrams) analysis reveals distinct association profile between the COVID-19 and control cohort (Fig. 1D). Within the COVID-19 cohort, we 
observed high positive correlations between the BIM and inflammatory CCs indicative of a brain injury mechanism triggered by systemic inflammation. While IL6 levels were not significantly higher in the COVID-19 cohort, as previous studies have found they trended higher in men. In our cohort, we observed IL6 to highly correlate with other CCs and BIMs (Fig. 1D). Several inflammatory CCs and notably MCP1, IP10, IL6, IL1Ra, IL10 and TNFa had more associations (Fig. 1D, COVID-19) with the BIMs suggesting that these CCs are the inflammatory markers driving brain injury.

\section{Severity and outcomes}

We examined the levels of BIMs, EIMs and CCs across COVID-19 severity (mild, moderate and severe). The levels of BIM were not significantly different across severity. However, one EIM (sICAM1) and two CCs (SCD40L and IL1RA) showed a near-significant trend of elevation in high severity (Fig. 2A). None of the BIMs or EIMs were predictive of functional outcomes at discharge. Two CCs, Eotaxin and MIP1b were significantly higher $(p<0.05)$ in subjects who proceeded to have poor outcomes (Fig. 2B). When adjusted for age and sex, only MIP1b was found to be independently associated with functional outcomes. MDC was significantly $(p<0.05)$ lower in those who proceeded to have poor outcomes. However, when controlled for age and sex in a multivariate logistic regression model, only MIP1b was associated with functional outcomes (Fig. 2B).

\section{Sex differences}

Among the BIMs, only the levels of NSE was significantly higher in men compared to women $(7.95 \pm 0.9$ vs. $22.9 \pm 5.8, p=0.01)$. BIMs including S100B, tau and MAP2 were higher in men; however, these differences were not statistically significant (Fig. 3A). The levels of the EIMs were not significantly different across men and women (data not shown). Four CCs were significantly higher in men compared to women: IL10 ( $35 \pm 10.2$ vs. $14.4 \pm 2.3, p=0.05)$, IL15 ( $5 \pm 1.1$ vs. $2.4 \pm 0.6, p=0.4$ ), IL8 $(32 \pm 9.2$ vs. $11.4 \pm 3.06, p=0.04)$ and MIP1a $(3.3 \pm 0.7$ vs. $1.4 \pm 0.4, p=0.4)$. Four CCs including MIP1b, FGP2, GM-CSF and IL6 were near significantly $(p<0.1)$ higher in men compared to women (Fig. 3B). The BIM-EIM-CC interaction profile reveal stark differences between men than women (Fig. 3C). Men had a higher number of correlations between the BIM-EIM-CC and the correlation profile was significantly different compared to women $(p<0.05$, Fisher's test). There were more interactions between the CCs and BIMs suggestive of a higher degree of inflammation induced brain injury in men. Additionally, in women, the EIMs (sICAM1 and SVCAM1) were not significantly correlated with the CCs or BIMs. However, in men, the EIMS were highly correlated with CCs and BIMs (Fig. 3C).

The expression levels of all the proteins (BIMs, CCs and EIMs) that were not significant $(p>0.05)$ across the groups (i.e., across control vs. COVID-19, across sex and across severity) are presented (see Additional file 1: Tables S1, S2 and S3).

\section{Discussion}

We investigated the profile of brain injury, endothelial injury and inflammatory markers after COVID19 disease. We found that levels of BIMs, specifically MAP2, NSE and S100 $\beta$ were significantly elevated in the acute phase of COVID-19. Two EIMs, sICAM1 and sCAM-1, were elevated after COVID-19 infection with robust interactions with the BIMs and IL6. Additionally, a host of inflammatory markers was elevated after acute COVID-19 infection. Interestingly we found men expressed significantly higher BIM levels than women suggesting a strong sex-dependent biological mechanism underlying the interactions between systemic inflammation, endothelial damage and brain injury after COVID19. Even among the proteins that were non-significant across the groups, there was a trend towards increased inflammatory and injury markers in COVID-19 subjects and in men compared to women.

The neuro-invasive and neurotropic nature of human coronaviruses $(\mathrm{HCoV})$ are known, and they may contribute to both short- and long-term neurological disorders [9-11]. The neuroinvasion of $\mathrm{HCoVs}$ confirmed by the presence of viral RNA in the human CNS adds credence to this hypothesis [30]. Acute neurologic and psychiatric complications have been reported after COVID-19 hospitalizations [2]. A wide range of neurological manifestations including ischemic stroke, intracerebral hemorrhage, hallucinations, encephalopathy, anosmia and ageusia have been described after SARS-CoV-2 infection [3-8]. Hyperemic and edematous brain tissue and neuronal death have been reported in autopsy cases after COVID-19 [31]. However, it is unknown if BIM are elevated in patients without overt neurological disease.

To investigate the effects of COVID-19 on BIMs, we examined systemic levels of six previously validated BIMs-MAP2, NSE, GFAP, S100B, Syndecan-1 and UCHL1. We found that MAP2, NSE and S100B were higher after COVID-19 indicative of brain injury after COVID-19. MAP-2 is a dendritic injury marker that is elevated in both the acute and chronic stages of brain injury [32]. This protein is typically localized in the dendritic regions and is involved in the promotion of microtubule synthesis and cross-linking with other compartments of the cytoskeleton. It is elevated after a traumatic brain injury and associated with long-term 

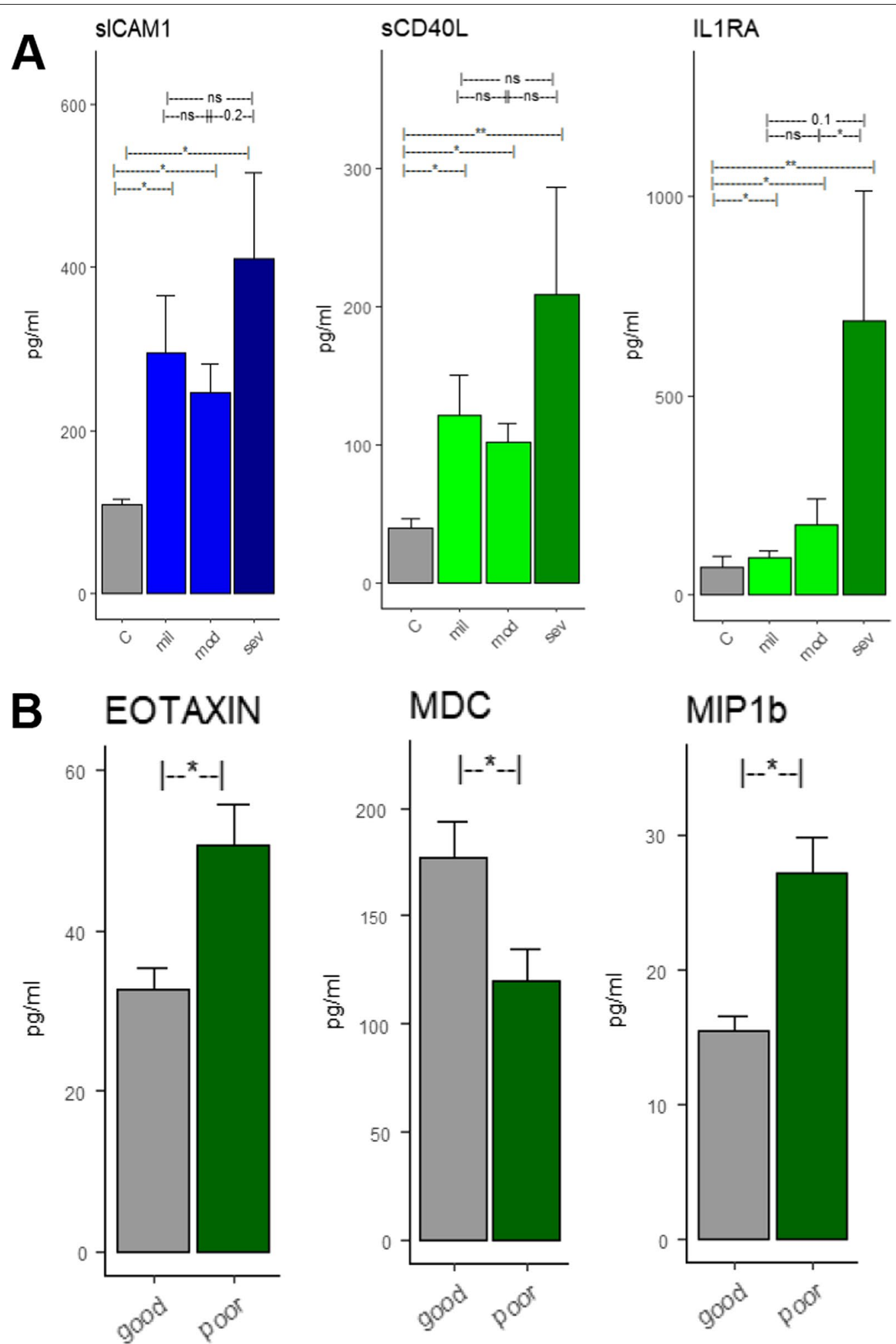

Fig. 2 Differences across severity and clinical outcomes. Differences across severity and clinical outcomes. A Difference in SICAM1, sCD40L and IL1RA across controls, mild, moderate and severe COVID groups. B Difference in CC levels across functional outcomes at discharge. EOTAXIN, MDC and MIP1b were significantly different across the good and the poor functional outcome groups. However, when adjusted for age and sex, only MIP1b was independently associated with outcomes 

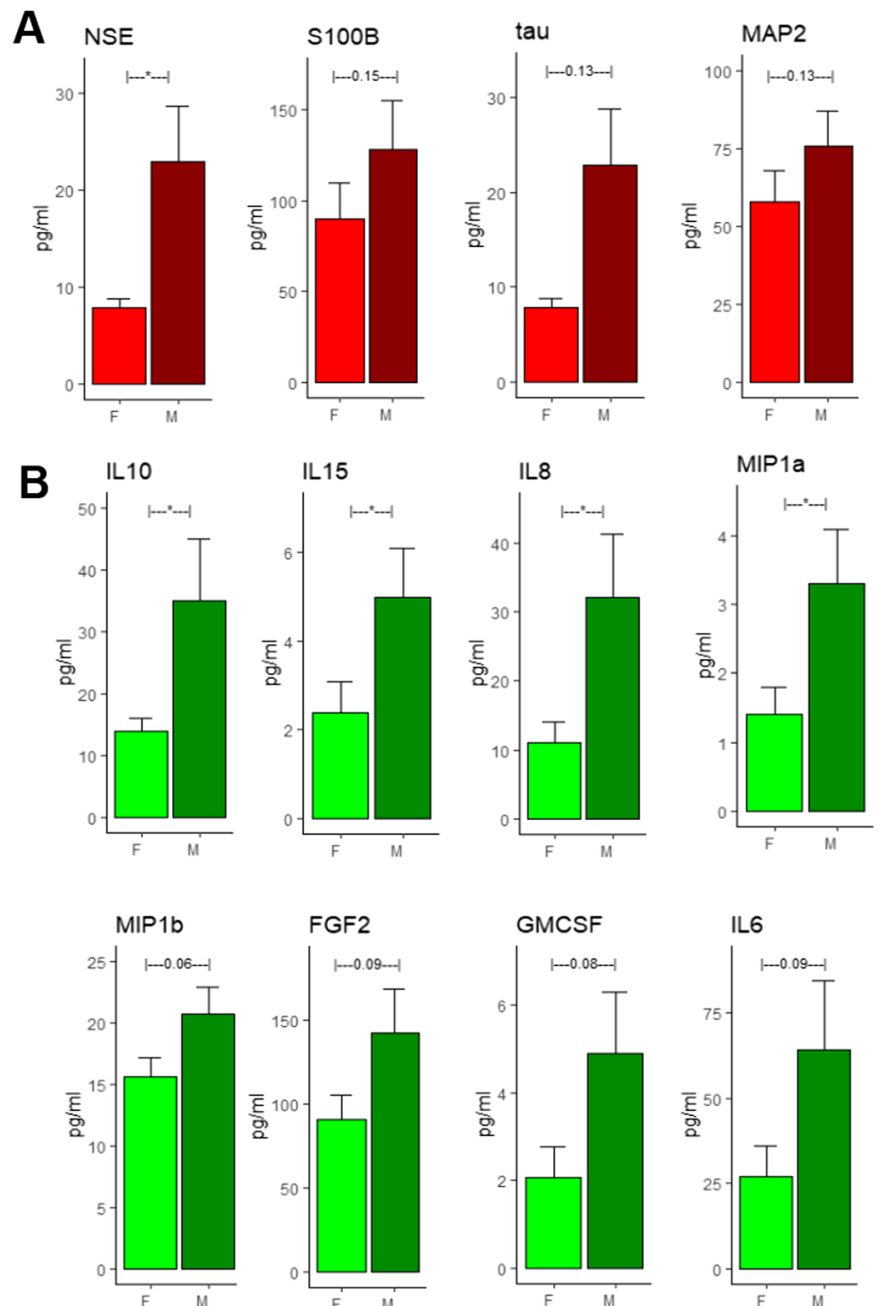
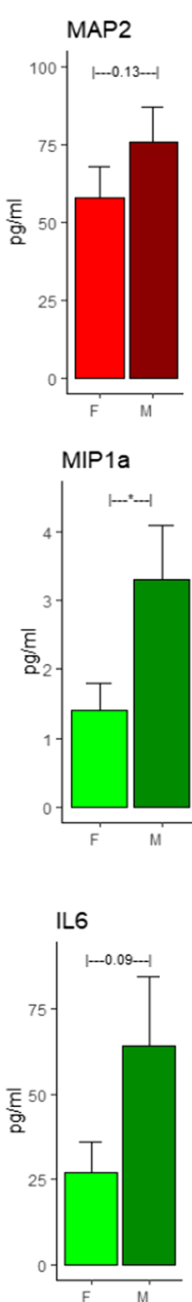

C Women
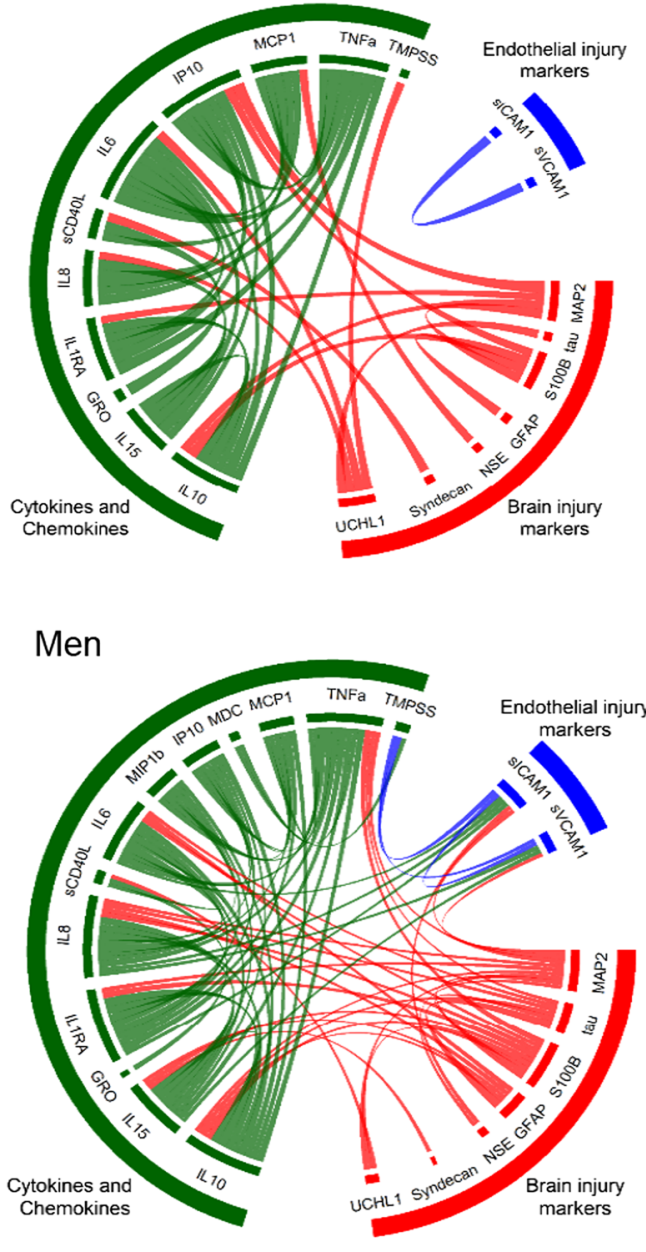

Fig. 3 Sex difference in BIM and CC profile across COVID-19 subjects. A NSE was significantly $(p<0.05)$ higher after COVID-19. S100b, tau and MAP2 were higher but differences were not statistically significant B IL10, IL15, IL8 and MIP1a were significantly $(p<0.05)$ in males compared to females. MIP1b, FGF2, GM-CSF and IL6 indicated a non-significant trend of being elevated in males. C The BIM-EM-CC interactions reveal that males have a much robust interactions compared to females. The results indicate that there was a robust association between the inflammatory-brain injury markers in 'males' compared to 'females'. Males had 75 significant $(p<0.05)$ associations between the markers compared to females who only had 39 significant associations indicating a higher overall inflammatory-mediated brain injury marker mechanisms in male compared to females. The similarity index (SI) between two matrices is 0.71 , a $p$-value $<0.0001$ using the random skewers method

outcomes [32]. NSE is an acidic protease present in neurons and neuroendocrine cells and is a useful indicator of neuronal damage [33]. S100B is a calcium-binding protein that is localized in astrocytic cytoplasm. It has been shown to be elevated systemically especially in traumatic brain injuries [34] and strokes [35]. Systemic levels of S100B are higher after traumatic brain injury and have been associated with poor long-term outcomes [36-38]. Increased levels of MAP2, NSE and S100B indicate neuronal and astrocytic injury after COVID-19 (Fig. 1A). Additionally, we found that BIMs (NSE, S100 $\beta$, tau, MAP2) were higher in men compared to women with COVID-19. It is not clear whether the elevations in BIMs overall and specifically in men have clinical relevance. We deliberately excluded samples from subjects with overt neurologic injury (i.e., traumatic brain injury, ischemic stroke, or hemorrhagic stroke) associated with COVID-19 disease. The elevated BIMs suggest that there is CNS involvement in acute COVID-19 and the clinical ramifications of this for chronic outcomes requires investigation.

Several pro-inflammatory cytokines including IL10, MCP1, TNFa, MDC, GRO, sCD40L, IL1Ra and IP10 were higher after COVID-19 compared to controls consistent 
with findings from several recent studies [39-41]. We also found that EIMs (sICAM-1 and sVCAM-1) were higher after COVID-19 infection (Fig. 1B). Endothelial cells express the ACE-2 receptor-the primary receptor and main route in intracellular entry for SARS-CoV-2 [42]. The blood-brain barrier (BBB) via the endothelial tight junctions is critical in maintaining cerebral homeostasis and $\mathrm{BBB}$ breakdown is implicated in variety of neurological diseases [43]. The BBB is compromised in COVID-19 and the SARS-CoV-2 spike protein can cross the BBB into brain tissue $[44,45]$. The endothelial dysfunction might be a direct result of the SARS-CoV-2 infection or due to a reaction to systemic inflammation triggered by the viral infection, or a combination of these factors. ICAM-1 is expressed in the vascular endothelium and is typically induced by IL-1b and TNF $\alpha$ [46]. It promotes leukocyte adhesion [47] and affects barrier function and vascular leakage [48]. sICAM-1 is the circulating form of ICAM- 1 and has been identified as a candidate marker of vascular inflammation [49]. Increased levels of sICAM-1 have been observed in cardiovascular disease including myocarditis [50] and inflammatory cardiomyopathy [51]. In addition to increased levels of SICAM-1, we found that TNF $\alpha$ was also elevated after COVID-19. In our data, the simultaneous elevation of the pro-inflammatory TNF $\alpha$ and sICAM-1 is suggestive of an inflammatory-mediated vascular injury rather than a virally triggered vascular injury (Fig. 1). In fact, a recent NIH funded post-mortem study examining the brain tissue of deceased COVID-19 subjects reported extensive inflammation and microvascular blood vessel damage, but it is not clear how SARS$\mathrm{CoV}-2$ is involved in the brain injury and neurological outcome [52]. The findings of this study suggest that the microvascular blood vessel damage is likely caused by the inflammatory response due to the infection, rather than the virus itself.

Interestingly, the anti-inflammatory marker IL10 was elevated in COVID-19 subjects. The elevation of IL10 could simply be a response to elevations in pro-inflammatory processes after COVID-19 infection in addition to other inflammatory markers. Alternatively, this could indicate the activation of immunosuppression mechanisms, which has been implicated in poor outcomes in brain injury. Elevation in IL10 supports the idea that early immune suppression may play a detrimental role in brain injury. Perhaps a dysregulation of the immune process in addition to overwhelming inflammation is the driver of pathophysiological processes [53]. IP-10 is shown to be involved in both pro-inflammatory and regulatory mechanisms and IP-10 signaling is a known promoter of T-cell recruitment and activation [54]. Two pathways involving IP-10: the restraining of IP-10 production by CCL5 [55] and the inhibition of regulatory $\mathrm{T}$-cell recruitment by
IP-10 [54], have been previously implicated in controlling inflammatory response and in neuro protection $[56,57]$. Another study has reported an increased level of IP10 and MCP1 as biomarkers after COVID-19 [58]. IP-10 can also inhibit endothelial recovery [59] and investigations in anti-IP10 antibody is underway to test to promote endothelial healing. A similar anti-IP10 antibody treatment has been suggested in COVID-19 patients with thrombotic events.

Neuronal injury after COVID-19 infection is documented and multiple mechanisms via of CNS damage including viral mediated injury, hyper-inflammation and tissue hypoxia due to respiratory failure have been purported. Direct CNS infection by SARS-CoV has been shown in humans and mice [11], and SARS-CoV-2 appears to have the potential to directly infect the brain and cerebral vasculature of humans. However, it is unclear whether the CNS damage is a result of the viral infection of due thrombotic microangiopathy and systemic hyper-inflammation. An alternate mechanism of neuronal injury includes CNS hypoxia due to respiratory failure. Our finding that severity of respiratory compromise did not correlate with BIM suggests the brain hypoxia is not the dominant cause for brain injury. On the other hand, there is increasing mechanistic evidence that neuronal injury is mediated primarily via hyperinflammation and endothelial dysfunction. A study examining neuronal injury after COVID-19 patients reported the presence CNS damage likely due to endothelial leakage [60]. This post-mortem study used investigated MRI scans of olfactory bulbs and brainstems in 16 COVID-19 patients and reported the presences of hyperintensity and hypointensity regions-which indicate the presence of inflammation and bleeding, respectively. They reported the presence of thin endothelial vessels and the presence of fibrinogen-a marker of BBB leakage. The hyperintensity regions were surrounded by $\mathrm{T}$ cells and microglia and the hypointensity regions contained both clotted and leaky blood vessels but no immune response. Additionally, they reported no evidence of SARS-CoV-2 infection in the brain tissue suggesting that the CNS injury was not caused by the direct viral attached in the brain, but rather due to microvascular blood vessel damage as the body's response to the virus. The authors also ruled out injury due to CNS hypoxia as the areas of damage were multifocal that are typically observed in strokes and other neuroinflammatory diseases. In another study, a SARS-CoV-2-mediated neuroinflammatory response was reported via investigating small cell clusters of early-activated macrophages in the olfactory epithelium [61]. An upregulation of (HLA)-DR on macrophages and increased levels of myeloid-driven inflammatory response (including an elevated levels of IL6, IL18, CCL2 
and sICAM-1 in the CSF) were reported. Furthermore, histopathological investigations indicated micro-thrombosis and acute brain infarcts in $18 \%$ of the subjects.

To elucidate the relationships between the BIMs, EIMs and systemic CCs in our cohort of subjects, we employed a bioinformatics visualization tool called the chord diagram and overlaid the significant correlations. In the COVID-19 cohort, the systemic CCs IL6, IL1Ra, IL10, TNFa, MCP1 and IP10 had high associations with the BIMs. Among the BIMs, MAP2 and S100B had the most associations with the systemic CCs (Fig. 1D). Particularly, the pro-inflammatory cytokines TNFa, IP10, IL1Ra and IL6 were strongly correlated with the MAP2 and S100B which were absent in the control group (Fig. 1). The relationship between brain injury and excessive systemic inflammation has previously been reported in other acute diseases [62-64]. The systemic inflammatory signals are purported to affect brain regions via either the humoral/ neural pathways (via a compromised $\mathrm{BBB}$ ) or via the activation of the vagus nerve [65-67]. For instance, systemic levels of TNFa have been reported to mediate brain injury processes after TBI and stroke [68] and inflammation in astrocytes [69]. Experimental models have shown that the deficiency of TNFa results in less cortical tissue loss after brain injury [70]. IL6 has also been shown to mediate brain injury. The strong association between the BIMs and the pro-inflammatory IL6 and TNFa in our cohort suggests the presence of inflammation-mediated neuronal injury mechanisms. Besides specific associations, there were relatively higher correlations between the BIMs and CCs in the COVID-19 cohort compared to controls suggest inflammation induced brain injury in COVID-19. It is known that inflammatory diseases are associated with neuronal injury (as in the case of encephalitis after sepsis [64]). Though our study design is nonmechanistic and direct causative mechanisms cannot be inferred, it is plausible that our findings, taken together with previous studies, support the view that the extensive cytokine activation by COVID-19 infection is the driver of endothelial injury that leads to neuronal and astrocytic injury in COVID-19.

Interestingly, the levels of BIMs were not different across clinical severity or clinical outcomes. sICAM1 and two CCs (sCD40L and IL1Ra) were higher in severe grade subjects (Fig. 2A), but this difference was not significant. Three CCs (Eotaxin, MDC and MIP1b) were different across clinical outcomes (Fig. 2B). After adjusting for age and sex, only MIP1b was independently associated with clinical outcomes. The lack of differences in BIMs levels across severity or clinical outcomes is likely a reflection of the time-point at which the samples were drawn rather than a pathophysiological mechanism of the disease process itself. Subject severity (mild, moderate or severe) was based on the course of hospital events (like requirement of high-flow cannula for extended time-period, ventilator, etc.) and not based on the symptoms that were observed at admission. Since we only used the first time-point for this study (obtained at $<48 \mathrm{~h}$ after admission)-which is around the time of admission, it is likely that these samples reflect a protein profile from a cohort of homogenous severity subjects. Additionally, the sample size of this study is relatively low and these observations need to be confirmed in another cohort with a larger sample size.

The long-term neurological effects of COVID-19 are increasingly recognized [71]. As more COVID19 patients are recovering from hospitalization, it is expected that many discharged patients will be affected with secondary complications and symptoms [72]. These symptoms are referred to as post-acute sequelae of COVID-19 or of SARS-CoV-2 (PASC) and include a host of medical symptoms that include functional, cognitive and psychiatric symptoms [73-75].

COVID-19 disproportionately affects men more severely compared to women. Preliminary studies have suggested that while the prevalence of infection is the same in men and women, men are more likely to be hospitalized, have a more severe disease course, and higher mortality than women [15]. We investigated differences in BIMs and CCs across men and women and we found that NSE was significantly elevated in men compared to women. Additionally, MAP2, tau and S100B showed trends towards elevated levels in men (Fig. 3A). Several CCs including IL15, IL8, MIP1b and IL10 were elevated in men compared to women (Fig. 3B) suggesting an increased inflammatory response in men. Men had a higher degree of inflammatory interactions (Fig. 3C) compared to women. Additionally, the interactions between the inflammatory markers (CCs) and the BIMs (red lines) were significantly higher in men indicating a higher degree of inflammation-mediated brain injury in men during the acute stages of COVID19 (Fig. 3C). Interestingly, the interactions between the EIMs and the BIMs/CCs were also higher in men compared to women (Fig. 3C). This suggests that men likely experience a higher degree of inflammation-triggered endotheliopathy - an important factor to be considered when developing treatment plans. A number of reasons have been postulated for the differential response across sex to COVID-19. Some studies have shown that men have a higher level of ACE2 receptors, used for SARS$\mathrm{CoV}-2$ virus entry [76]. Others have postulated that the differences are due to differences in lifestyle factors and co-morbidities between men and women [76, 77]. Given our small sample size, we are not powered to determine if these differences in BIMs translate into worse neurologic outcomes. Our finding add to the studies that show 
men have a more robust humeral response to COVID19 infection. We show that the inflammatory response is more tightly associated with endothelial disfunction and brain injury in men compared to women emphasize the sex-specific reactions to COVID-19. Despite the sex differences seen in COVID-19, treatment protocols and interventional studies have largely ignored these differences in favor of treatment regiments that are uniform across sexes. As we now transition into examining the long-term consequences of COVID-19, these sex differences in connections between inflammation, endothelial injury and brain injury need to be addressed for identification of successful therapeutics. Future studies investigating sex differences in the immune response to COVID-19 are required.

\section{Limitations}

Our study has several limitations. First, we included samples only from the first-available time-point after hospitalization. Typically, the measurements from the first-available time-point are a reflection of the patient's status during the earlier course of disease progress. However, this time-point is also the most accurate, as in our hospital all COVID-19 patients were administered dexamethasone, which could have a confounding effect on the measurements. Second, we have not related the observed BIMs with any long-term clinical outcomes. Third, out study has a relatively low sample size. We are underpowered to detect the effect of any co-morbidities on the neuronal injury. Finally, this is an observational study and the study design only allows for correlational associations. Further studies are required to establish direct causative mechanisms.

\section{Conclusion}

Our findings suggest that immediately after COVID-19 hospitalization, BIMs were higher than matched controls. The levels of EIMs and CCs were also higher. The CCs were highly correlated with BIMs and EIMs, suggesting inflammatory-driven endothelial dysfunction and brain injury in the acute phase of COVID-19. Further studies are required to clarify the exact mechanisms how COVID-19 induces inflammation and brain injury, sexspecific differences and whether these early increase in injury markers are associated with long-term neurologic outcomes.

\footnotetext{
Abbreviations

BIM: Brain injury markers; CC: Cytokines/chemokines; EIM: Endothelial injury markers; mRS: Modified Rankin Score; BBB: Blood-brain barrier; SARS-CoV: Severe acute respiratory syndrome-associated coronavirus; UCHL-1: Ubiquitin carboxy-terminal hydrolase L1; MAP2: Microtubule-associated protein 2; GFAP: Glial fibrillary acidic protein; NSE: Neuron-specific enolase; sICAM1: Soluble
}

intercellular adhesion molecule-1; SVCAM1: Soluble vascular cell adhesion molecule 1.

\section{Supplementary Information}

The online version contains supplementary material available at https://doi. org/10.1186/s12974-021-02323-8.

Additional file 1. Protein differences (non-significant) in COVID-19 vs Controls, sex (within COVID-19 subjects) and across COVID-19 severity.

\section{Acknowledgements}

The authors thank all the health care workers and the research personnel who tirelessly worked in the care and the follow-up of these patients. We thank The Biorepository of Neurological Diseases team for their efforts in collecting the samples.

\section{Authors' contributions}

$J S, L M, F L, P D$ and $H A C$ were involved in the conception and design of the study. JS was involved in analysis of data. EP, GDC, SNH, DM, HA, ASP, AA, FL and SJ and were involved in the acquisition of data. JS, EP, EK, SC, AMG, FL, PD, LM and HAC contributed substantially in drafting the manuscript and figures. All authors read and approved the final manuscript.

\section{Funding}

This work was partly supported by a donation from the Huffington Foundation to Dr. Louise D. McCullough.

\section{Availability of data and materials}

All data generated and analyzed during this study are available from the corresponding author on reasonable request.

\section{Declarations}

Ethics approval and consent to participate

This study adhered to the ethical guidelines and was approved by the 'Institutional Review Board' (IRB No: HSC -MH-17-0452) at The University of Texas Health Science Center at Houston, Houston, Texas.

\section{Consent for publication}

The authors provide consent for publication.

\section{Competing interests}

The authors declare that they have no conflict of interest.

\section{Author details}

${ }^{1}$ Departent of Neurosurgery, McGovern Medical School, The University of Texas Health Science Center at Houston, 6431 Fannin St, Houston, TX 77030 USA. ${ }^{2}$ Department of Neurobiology, McGovern Medical School, The University of Texas Health Science Center at Houston, Houston, TX 77030, USA. ${ }^{3}$ Johns Hopkins University School of Medicine, Baltimore, MD 21205, USA. ${ }^{4}$ Department of Neurology, McGovern Medical School, The University of Texas Health Science Center at Houston, Houston, TX 77030, USA.

Received: 7 July 2021 Accepted: 14 November 2021

Published online: 27 November 2021

\footnotetext{
References

1. Ellul MA, Benjamin L, Singh B, Lant S, Michael BD, Easton A, et al. Neurological associations of COVID-19. Lancet Neurol. 2020;19:767-83.

2. Varatharaj A, Thomas N, Ellul MA, Davies NWS, PollakTA, Tenorio EL, et al. Neurological and neuropsychiatric complications of COVID-19 in 153 patients: a UK-wide surveillance study. Lancet Psychiatry. 2020;7:875-82.

3. Lee Y, Min P, Lee S, Kim S-W. Prevalence and duration of acute loss of smell or taste in COVID-19 patients. J Korean Med Sci [Internet]. 2020. https://www.ncbi.nlm.nih.gov/pmc/articles/PMC7211515/.
} 
4. Russell B, Moss C, Rigg A, Hopkins C, Papa S, Hemelrijck MV. Anosmia and ageusia are emerging as symptoms in patients with COVID-19: what does the current evidence say? [Internet]. 2020. http://ecancer.org/en/journal/ editorial/98-anosmia-and-ageusia-are-emerging-as-symptoms-in-patie nts-with-covid-19-what-does-the-current-evidence-say.

5. Vaira LA, Salzano G, Deiana G, De Riu G. Anosmia and Ageusia: Common Findings in COVID-19 Patients. Laryngoscope [Internet]. 2020. https:// www.ncbi.nlm.nih.gov/pmc/articles/PMC7228304/.

6. Large-Vessel Stroke as a Presenting Feature of Covid-19 in the Young | NEJM [lnternet]. https://doi.org/10.1056/NEJMc2009787.

7. Montalvan V, Lee J, Bueso T, De Toledo J, Rivas K. Neurological manifestations of COVID-19 and other coronavirus infections: a systematic review. Clin Neurol Neurosurg. 2020;194:105921.

8. Asadi-Pooya AA, Simani L. Central nervous system manifestations of COVID-19: a systematic review. J Neurol Sci. 2020;413:116832.

9. Desforges M, Le Coupanec A, Dubeau P, Bourgouin A, Lajoie L, Dubé M, et al. Human coronaviruses and other respiratory viruses: underestimated opportunistic pathogens of the central nervous system? Viruses [Internet]. 2019. https://www.ncbi.nlm.nih.gov/pmc/articles/PMC7020001/.

10. Yeh EA, Collins A, Cohen ME, Duffner PK, Faden H. Detection of coronavirus in the central nervous system of a child with acute disseminated encephalomyelitis. Pediatrics. 2004;113:e73-6.

11. Lau K-K, Yu W-C, Chu C-M, Lau S-T, Sheng B, Yuen K-Y. Possible central nervous system infection by SARS coronavirus. Emerg Infect Dis. 2004;10:342-4.

12. Xu J, Zhong S, Liu J, Li L, Li Y, Wu X, et al. Detection of severe acute respiratory syndrome coronavirus in the brain: potential role of the chemokine mig in pathogenesis. Clin Infect Dis. 2005;41:1089-96.

13. Gu J, Gong E, Zhang B, Zheng J, Gao Z, Zhong Y, et al. Multiple organ infection and the pathogenesis of SARS. J Exp Med. 2005;202:415-24.

14. Gebhard C, Regitz-Zagrosek V, Neuhauser HK, Morgan R, Klein SL. Impact of sex and gender on COVID-19 outcomes in Europe. Biol Sex Differ [Internet]. 2020. https://www.ncbi.nlm.nih.gov/pmc/articles/PMC72 47289/.

15. Jin J-M, Bai P, He W, Wu F, Liu X-F, Han D-M, et al. Gender differences in patients with COVID-19: focus on severity and mortality. Front Public Health [Internet]. 2020. https://www.ncbi.nlm.nih.gov/pmc/articles/ PMC7201103/.

16. Scully EP, Haverfield J, Ursin RL, Tannenbaum C, Klein SL. Considering how biological sex impacts immune responses and COVID-19 outcomes. Nat Rev Immunol. 2020;20:442-7.

17. Ahnstedt $H$, McCullough LD. The impact of sex and age on T cell immunity and ischemic stroke outcomes. Cell Immunol. 2019;345:103960.

18. Limpruttidham N, Mitchell BI, Kallianpur KJ, Nakamoto BK, Souza SA, Shiramizu B, et al. S100B and its association with HIV-associated neurocognitive disorders. J Neurovirol. 2019;25:899-900.

19. Sporer B, Missler U, Magerkurth O, Koedel U, Wiesmann M, Pfister HW. Evaluation of CSF glial fibrillary acidic protein (GFAP) as a putative marker for HIV-associated dementia. Infection. 2004;32:20-3.

20. Levine AJ, Soontornniyomkij V, Achim CL, Masliah E, Gelman BB, Sinsheimer JS, et al. Multilevel analysis of neuropathogenesis of neurocognitive impairment in HIV. J Neurovirol. 2016;22:431-41.

21. Tiainen $M$, Roine $R O$, Pettilä $V$, Takkunen $O$. Serum neuron-specific enolase and S-100B protein in cardiac arrest patients treated with hypothermia. Stroke. 2003;34:2881-6.

22. Helwig K, Seeger F, Hölschermann H, Lischke V, Gerriets T, Niessner M, et al. Elevated serum glial fibrillary acidic protein (GFAP) is associated with poor functional outcome after cardiopulmonary resuscitation. Neurocrit Care. 2017;27:68-74.

23. Ebner F, Moseby-Knappe M, Mattsson-Carlgren N, Lilja G, Dragancea I, Undén J, et al. Serum GFAP and UCH-L1 for the prediction of neurological outcome in comatose cardiac arrest patients. Resuscitation. 2020;154:61-8.

24. Wu L, Feng Q, Ai M-L, Deng S, Liu Z-Y, Huang L, et al. The dynamic change of serum S100B levels from day 1 to day 3 is more associated with sepsisassociated encephalopathy. Sci Rep. 2020;10:7718.

25. Modified Rankin Score (mRS) [Internet]. https://manual.jointcommission. org/releases/TJC2018A/DataElem0569.html.

26. Box GEP, Cox DR. An analysis of transformations. J Royal Stat Soc Series B (Methodological). 1964;26:211-52.

27. Rohlf FJ. The method of random skewers. Evol Biol. 2017;44:542-50.
28. Gu Z, Gu L, Eils R, Schlesner M, Brors B. Circlize implements and enhances circular visualization in R. Bioinformatics. 2014;30:2811-2.

29. Modified Rankin Scale for Neurologic Disability —MDCalc [Internet] https://www.mdcalc.com/modified-rankin-scale-neurologic-disability.

30. Arbour N, Day R, Newcombe J, Talbot PJ. Neuroinvasion by human respiratory coronaviruses. J Virol. 2000;74:8913-21.

31. Mao L, Jin H, Wang M, Hu Y, Chen S, He Q, et al. Neurologic manifestations of hospitalized patients with Coronavirus Disease 2019 in Wuhan, China. JAMA Neurol. 2020;77:683-90.

32. Mondello S, Gabrielli A, Catani S, D'Ippolito M, Jeromin A, Ciaramella A, et al. Increased levels of serum MAP-2 at 6-months correlate with improved outcome in survivors of severe traumatic brain injury. Brain Inj. 2012;26:1629-35.

33. Thelin EP, Jeppsson E, Frostell A, Svensson M, Mondello S, Bellander $B-M$, et al. Utility of neuron-specific enolase in traumatic brain injury; relations to S100B levels, outcome, and extracranial injury severity. Crit Care. 2016;20:285.

34. Thelin EP, Nelson DW, Bellander B-M. A review of the clinical utility of serum $\mathrm{S} 100 \mathrm{~B}$ protein levels in the assessment of traumatic brain injury. Acta Neurochir (Wien). 2017;159:209-25.

35. Manfred H, Pieter V, Wunderlich Michael T, de Bruijn Chris HMM, Lamers Karl JB. Release of Glial tissue-specific proteins after acute stroke. Stroke. 2000;31:2670-7.

36. Goyal A, Failla MD, Niyonkuru C, Amin K, Fabio A, Berger RP, et al. S100b as a prognostic biomarker in outcome prediction for patients with severe traumatic brain injury. J Neurotrauma. 2013;30:946-57.

37. Mercier E, Boutin A, Lauzier F, Fergusson DA, Simard J-F, Zarychanski R, et al. Predictive value of S-100 $\beta$ protein for prognosis in patients with moderate and severe traumatic brain injury: systematic review and meta-analysis. BMJ. 2013;346:f1757.

38. Thelin EP, Johannesson L, Nelson D, Bellander B-M. S100B is an important outcome predictor in traumatic brain injury. J Neurotrauma. 2013;30:519-28.

39. Tay MZ, Poh CM, Rénia L, MacAry PA, Ng LFP. The trinity of COVID19: immunity, inflammation and intervention. Nat Rev Immunol. 2020;20:363-74.

40. Zhao Y, Qin L, Zhang P, Li K, Liang L, Sun J, et al. Longitudinal COVID-19 profiling associates IL-1RA and IL-10 with disease severity and RANTES with mild disease. JCI Insight. 2020;5:e139834.

41. Lu L, Zhang H, Dauphars DJ, He Y-W. A potential role of interleukin 10 in COVID-19 pathogenesis. Trends Immunol. 2021;42:3-5.

42. Dalan R, Bornstein SR, El-Armouche A, Rodionov RN, Markov A, Wielockx B, et al. The ACE-2 in COVID-19: Foe or Friend? Horm Metab Res. 2020;52:257-63.

43. Weiss N, Miller F, Cazaubon S, Couraud P-O. The blood-brain barrier in brain homeostasis and neurological diseases. Biochim Biophys Acta. 2009; 1788:842-57.

44. Buzhdygan TP, DeOre BJ, Baldwin-Leclair A, Bullock TA, McGary HM, Khan JA, et al. The SARS-CoV-2 spike protein alters barrier function in 2D static and 3D microfluidic in-vitro models of the human bloodbrain barrier. Neurobiol Dis. 2020;146:105131.

45. Rhea EM, Logsdon AF, Hansen KM, Williams LM, Reed MJ, Baumann KK, et al. The S1 protein of SARS-CoV-2 crosses the blood-brain barrier in mice. Nat Neurosci. 2021;24:368-78.

46. Myers CL, Wertheimer SJ, Schembri-King J, Parks T, Wallace RW. Induction of ICAM-1 by TNF-alpha, IL-1 beta, and LPS in human endothelial cells after downregulation of PKC. Am J Physiol. 1992;263:C767-772.

47. Long EO. Intercellular adhesion molecule 1 (ICAM-1): getting a Grip on Leukocyte Adhesion. J Immunol [Internet]. 2011. https://www.ncbi. nlm.nih.gov/pmc/articles/PMC3860744/.

48. Clark PR, Manes TD, Pober JS, Kluger MS. Increased ICAM-1 expression causes endothelial cell leakiness, cytoskeletal reorganization and junctional alterations. J Investig Dermatol. 2007;127:762-74.

49. Witkowska AM. Soluble ICAM-1: a marker of vascular inflammation and lifestyle. Cytokine. 2005;31:127-34.

50. Toyozaki T, Saito T, Takano H, Yorimitsu K, Kobayashi S, Ichikawa H, et al. Expression of intercellular adhesion molecule-1 on cardiac myocytes for myocarditis before and during immunosuppressive therapy. Am J Cardiol. 1993;72:441-4. 
51. Noutsias M, Hohmann C, Pauschinger M, Schwimmbeck P-L, Ostermann $\mathrm{K}$, Rode $\mathrm{U}$, et al. sICAM-1 correlates with myocardial ICAM-1 expression in dilated cardiomyopathy. Int J Cardiol. 2003;91:153-61.

52. NIH study uncovers blood vessel damage and inflammation in COVID-19 patients' brains but no infection [Internet]. National Institutes of Health (NIH). 2020. https://www.nih.gov/news-events/news-releases/nih-studyuncovers-blood-vessel-damage-inflammation-covid-19-patients-brainsno-infection.

53. Sarrafzadeh A, Schlenk F, Gericke C, Vajkoczy P. Relevance of cerebral interleukin-6 after aneurysmal subarachnoid hemorrhage. Neurocrit Care. 2010;13:339-46.

54. Potteaux S, Combadière C, Esposito B, Lecureuil C, Ait-Oufella H, Merval $\mathrm{R}$, et al. Role of bone marrow-derived CC-chemokine receptor 5 in the development of atherosclerosis of low-density lipoprotein receptor knockout mice. Arterioscler Thromb Vasc Biol. 2006;26:1858-63.

55. Menten P, Wuyts A, Van Damme J. Macrophage inflammatory protein-1. Cytokine Growth Factor Rev. 2002;13:455-81.

56. Bartholomäus I, Kawakami N, Odoardi F, Schläger C, Miljkovic D, Ellwart $\mathrm{JW}$, et al. Effector T cell interactions with meningeal vascular structures in nascent autoimmune CNS lesions. Nature. 2009:462:94-8.

57. Shichita T, Sugiyama Y, Ooboshi H, Sugimori H, Nakagawa R, Takada I, et al. Pivotal role of cerebral interleukin-17-producing gammadeltaT cells in the delayed phase of ischemic brain injury. Nat Med. 2009;15:946-50.

58. IP-10 and MCP-1 as biomarkers associated with disease severity of COVID-19 | Molecular Medicine | Full Text [Internet]. https://doi.org/10. 1186/s10020-020-00230-x.

59. Lupieri A, Smirnova NF, Solinhac R, Malet N, Benamar M, Saoudi A, et al. Smooth muscle cells-derived CXCL10 prevents endothelial healing through PI3KY-dependent T cells response. Cardiovasc Res. 2020;116:438-49.

60. Microvascular Injury in the Brains of Patients with Covid-19| NEJM [Internet]. https://doi.org/10.1056/NEJMc2033369.

61. Meinhardt J, Radke J, Dittmayer C, Franz J, Thomas C, Mothes R, et al. Olfactory transmucosal SARS-CoV-2 invasion as a port of central nervous system entry in individuals with COVID-19. Nat Neurosci. 2020;1-8.

62. Lu J, Goh SJ, Tng PYL, Deng YY, Ling E-A, Moochhala S. Systemic inflammatory response following acute traumatic brain injury. Front Biosci (Landmark Ed). 2009;14:3795-813.

63. Savarraj J, Parsha K, Hergenroeder G, Ahn S, Chang TR, Kim DH, et al. Early brain injury associated with systemic inflammation after subarachnoid hemorrhage. Neurocrit Care. 2017;

64. Ren C, Yao R, Zhang H, Feng Y, Yao Y. Sepsis-associated encephalopathy: a vicious cycle of immunosuppression. J Neuroinflammation. 2020;17:14.

65. Dantzer R, Konsman JP, Bluthé RM, Kelley KW. Neural and humoral pathways of communication from the immune system to the brain: parallel or convergent? Auton Neurosci. 2000;85:60-5.

66. Dantzer R, Wollman EE. Relationships between the brain and the immune system. J Soc Biol. 2003;197:81-8.

67. Dantzer R. Cytokine-induced sickness behavior: where do we stand? Brain Behav Immun. 2001;15:7-24

68. Tuttolomondo A, Pecoraro R, Pinto A. Studies of selective TNF inhibitors in the treatment of brain injury from stroke and trauma: a review of the evidence to date. DDDT Dove Press. 2014;8:2221-39.

69. Hyvärinen T, Hagman S, Ristola M, Sukki L, Veijula K, Kreutzer J, et al. Co-stimulation with $\mathrm{L}-1 \beta$ and TNF- $\alpha$ induces an inflammatory reactive astrocyte phenotype with neurosupportive characteristics in a human pluripotent stem cell model system. Sci Rep. 2019;9:16944.

70. Scherbel U, Raghupathi R, Nakamura M, Saatman KE, Trojanowski JQ, Neugebauer $E$, et al. Differential acute and chronic responses of tumor necrosis factor-deficient mice to experimental brain injury. PNAS Natl Acad Sci. 1999;96:8721-6.

71. Nath A. Long-Haul COVID. Neurology. Wolters Kluwer Health, Inc. on behalf of the American Academy of Neurology; 2020;95:559-60.

72. NIH launches new initiative to study "Long COVID" [Internet]. National Institutes of Health (NIH). 2021. https://www.nih.gov/about-nih/ who-we-are/nih-director/statements/nih-launches-new-initi ative-study-long-covid.

73. Yelin D, Wirtheim E, Vetter P, Kalil AC, Bruchfeld J, Runold M, et al. Longterm consequences of COVID-19: research needs. Lancet Infect Dis Elsevier. 2020;20:1115-7.

74. Nath A. Long-Haul COVID. Neurology. 2020;95:559-60.
75. Martin S, Miyake E. Long Covid: quantitative and qualitative analyses of online Long Haulers' experiences, emotions and practices in the UK. 2020. https://europepmc.org/article/ppr/ppr221609.

76. Coronavirus: Why Men are More Vulnerable to Covid-19 Than Women? [Internet]. https://www.ncbi.n/m.nih.gov/pmc/articles/PMC7271824/.

77. Griffith DM. Men and COVID-19: a biopsychosocial approach to understanding sex differences in mortality and recommendations for practice and policy interventions. Prev Chronic Dis [Internet]. 2020;17. https:// www.cdc.gov/pcd/issues/2020/20_0247.htm.

\section{Publisher's Note}

Springer Nature remains neutral with regard to jurisdictional claims in published maps and institutional affiliations.

Ready to submit your research? Choose BMC and benefit from:

- fast, convenient online submission

- thorough peer review by experienced researchers in your field

- rapid publication on acceptance

- support for research data, including large and complex data types

- gold Open Access which fosters wider collaboration and increased citations

- maximum visibility for your research: over 100M website views per year

At BMC, research is always in progress.

Learn more biomedcentral.com/submissions 